\title{
Research on Cutting Mechanism and Cutting Force Theoretical Model Based on Indentation Fracture Mechanics in Turning Lithium Disilicate Glass
}

Lianjie Ma ( $\nabla$ mlj@mail.neu.edu.cn )

Northeastern University - Qinhuangdao Campus https://orcid.org/0000-0003-4952-0341

Kunjie Yan

Northeastern University, Shenyang

Shuhuai Wang

Northeastern University, Shenyang

Hongshuang Li

Northeastern University, Shenyang

Jing Jia

Northeastern University, Shenyang

\section{Research Article}

Keywords: Theoretical model of cutting force, Crack system, Cutter parameters, Energy conservation principle, Lithium disilicate glass

Posted Date: June 4th, 2021

DOI: https://doi.org/10.21203/rs.3.rs-557493/v1

License: (c) (i) This work is licensed under a Creative Commons Attribution 4.0 International License. Read Full License 


\title{
Research on Cutting Mechanism and Cutting Force
}

\section{Theoretical Model Based on Indentation Fracture Mechanics}

\section{in Turning Lithium Disilicate Glass}

\author{
Lianjie Ma ${ }^{1,2, a} \cdot$ Kunjie Yan ${ }^{1} \cdot$ Shuhuai Wang ${ }^{1} \cdot$ Hongshuang $\mathrm{Li}^{1} \cdot \mathrm{Jing} \mathrm{Jia}^{1}$ \\ 1. School of Mechanical Engineering and Automation, Northeastern University, Shenyang, Liaoning 110819, China \\ 2. School of Control Engineering, Northeastern University at Qinhuangdao, Qinhuangdao, Hebei 066004, China
}

\begin{abstract}
Based on the theory of energy transfer, this study analyzes the fracture mechanism of machinable ceramics, and establishes the machinable ceramic toolworkpiece-chip coupling cutting force model. Research on the brittleness removal of hard and brittle materials by indentation test. The pre-existing defects on the surface of the workpiece are subjected to tensile stress. With continuous loading on a certain point on the surface of the workpiece, the cracks begin to sprout and expand; when the critical load is loaded, the cracks become unstable; with the load continues, the conical cracks expand in an unstable state. The Hertz crack system is obtained at the end of the indentation. The crack system model is established based on indentation fracture mechanics. According to Griffith energy balance theory, different types of energy models of the crack system are established. The theoretical model of cutting force is established according to the principle of energy conservation, the tool input is equal to the energy change between crack systems. The results of turning experiments show that the main cutting force increases with the increase of the radius of the tool nose, and decreases with the increase of the tool rake angle. The calculated values of the theoretical model of cutting force in turning of hard and brittle materials are basically consistent with the experimental values.
\end{abstract}

Key words: Theoretical model of cutting force - Crack system - Cutter parameters Energy conservation principle $\cdot$ Lithium disilicate glass;

\section{Introduction}

Cutting force is one of the most critical parameters in the cutting process, and it plays an essential role in predicting machining quality and controlling machining accuracy ${ }^{[1]}$. The existing theoretical model of turning force only considers the influence of three factors of cutting parameters, the removal mechanism is not mature, and the theoretical model needs to be improved. It is of great significance to study the influence factors of cutting force and material removal mechanism and establish the cutting force model of hard and brittle materials for high precision and highefficiency machining of machinable ceramics ${ }^{[2]}$.

The cutting force model can be used to predict and control the deformation of hard and brittle materials, optimize the process parameters and provide a theoretical basis for physical simulation of the machining process ${ }^{[3]}$. Brittle materials have complex removal mechanisms of toughness and brittleness. Therefore, the traditional force model is mainly aimed at metal materials and can not be completely applied to the force prediction of brittle materials. At present, the theoretical models of cutting force for hard and brittle materials are mainly concentrated in milling, grinding, and drilling. $\mathrm{Ma}^{[5]}$ et al. proposed a continuous fracture mechanism for brittle materials. They

\footnotetext{
a Corresponding author.

E-mail address: mlj@mail.neu.edu.cn (L. Ma).
} 
established a theoretical model of continuous fracture mechanism because isolated cracks can only reflect a single micro-scale fracture behavior and are incompatible with the constant cutting process. Zhang ${ }^{[6]}$ established a mathematical model of cutting force in rotary ultrasonic end milling of brittle materials under the assumption that removing brittle materials is mainly the removal of brittle fracture. Yang ${ }^{[7]}$ carried out an ultrasonic vibration-assisted grinding experiment on zirconia ceramics and found that the load increase would change the material removal form from plastic removal to brittle removal. Combined with kinematics analysis, a prediction model of grinding force was established. By analyzing the brittle fracture removal mechanism of hard and brittle materials and the characteristics of rotary ultrasonic machining, Zhang Chenglong et al. ${ }^{[8]}$ determined the cutting time, cutting depth, cutting speed, cutting track length of a single abrasive particle during rotary ultrasonic machining, and established a mathematical prediction model of cutting force for drilling hard and brittle materials constant feed rate of rotary ultrasonic. Cheng $^{[9]}$ et al. found the cutting force model by using the least square method to fit the data through many turning experiments and concluded that the greater the cutting force, the more serious the tool wear would be.

Hard and brittle materials have physical characteristics such as high hardness, strength, and wear resistance. During turning, the cutting depth is usually less than the radius of the tip arc. So the turning process can be regarded as the blunt head to the material extrusion fracture. The head presses into the workpiece by force and cracks. The crack length calculates according to Hertz's theory. Based on the law of conservation of energy, the energy change of the crack system is equal to the tool input; it establishes the theoretical model of cutting force. Experiments of lithium disilicate glass-ceramics verified the accuracy of the model.

\section{Removing mechanism of brittle fracture in turning lithium disilicate glass}

\subsection{Crack length}

As shown in Fig. 1, the indentation test of the blunt head shows that the defectson the surface of the prefabricated workpiece are subjected to tensile stress. With continuous loading at a certain point on the workpiece surface, the crack begins to germinate. It propagates downward along the direction of the fastest reduction of stress in the tensile stress field. When loaded to a critical load, the ring crack becomes unstable. With the continuous loading, the conical crack expands in an unstable state. When the indentation is over, Hertz crack is obtained. The crack length is: ${ }^{[10]}$. The crack length is:

$$
c_{1}=\left(\chi P / K_{\mathrm{p}}\right)^{\frac{2}{3}}
$$

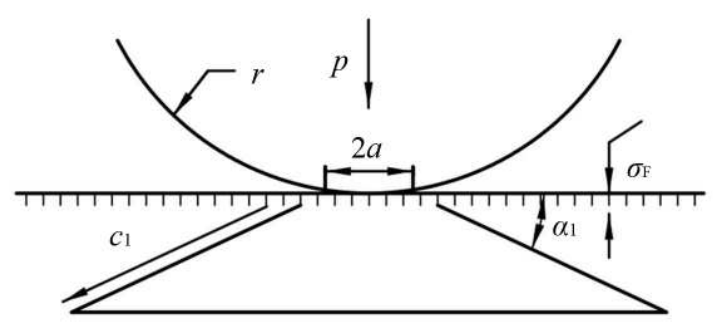

Fig. 1 .Conical crack system of lithium disilicate ceramics In Eq. (1), $c_{1}$ is the crack length in the first stage of large-scale stress failure, $\chi$ is the parameter, $P$ is the contact pressure, and $K_{\mathrm{p}}$ is the fracture toughness.

According to Hertz's elastic analysis, the contact pressure increases monotonically with the increase of the contact circle ${ }^{[11]}$.The relationship between the contact pressure and the radius of the tool-tip arc is:

$$
P=[3 E /(4 \pi k)](a / r)\left(\pi a^{2}\right)
$$

Where, $\quad k=(9 / 16)\left[\left(1-\mathrm{v}_{2}\right)+\left(1-\mathrm{v}_{\mathrm{s} 2}\right) E / E_{\mathrm{s}}\right] \quad$ is a dimensionless factor of 1 . Where $E$ and $v$ are the style modulus and Poisson's ratio of the elastic half-space, $E_{\mathrm{s}}$ and $v_{\mathrm{s}}$ are the style modulus and Poisson's ratio of the spherical indenter. 
According to Griffith principle, when the fracture occurs spontaneously, $\sigma_{\mathrm{A}}=\sigma_{\mathrm{F}}=\sigma_{\mathrm{I}}$ can obtain the characteristic material parameters of the material:

$$
T_{0}=\xi(E / H)^{\frac{1}{2}} P / C_{1}^{\frac{3}{2}}
$$

when $\mathrm{c}=\mathrm{c} 1, K \mathrm{p}=T_{0}$, combining Eqs. (1) and (3). The parameter $\chi$ is calculated as:

$$
\chi=\xi(E / H)^{\frac{1}{2}}
$$

Substituting Eqs. (2), (3) and (4) into Eq (1), obtains the fracture length of the first segment of the crack as:

$$
c_{1}=\frac{\left(4 \pi a^{3} \xi\right)^{\frac{2}{3}}(E / H)^{\frac{1}{3}}}{(3 r)^{\frac{2}{3}}}
$$

\subsection{Deflection Angle of crack system}

Crushing and fracture is the primary method for removing hard and brittle materials, which includes large-scale fracture and small-scale fracture. As shown in Fig.3, in the process of turning, large-scale fracture and small-scale fracture are carried out alternately. By analyzing the turning mechanism and observation of the experimental phenomenon, it is found that in the turning process of zirconia, the material removal is mainly by large-scale fracture removal. In the cutting process, the tool will not contact the machined surface, and the pits generated by large-scale fracture will remain on the machined surface. By observing the morphology of the craters, the crack growth Angle $\alpha_{1}$ in the first stage can be obtained, and then the crack deflection Angle and crack growth Angle $\alpha_{2}$ in the second stage can be obtained.

In Eq. (6), the inert strength is pre-determined by the defect distribution in the workpiece.

$$
\sigma_{\mathrm{I}}=K_{\mathrm{I}} / \psi c_{1}^{\frac{1}{2}}
$$

Where $\psi=2$ is a dimensionless coefficient.

Brittle cracks always tend to propagate in a direction that minimizes the shear load. As shown in Fig.2, among the three types, type I (open type) expands under the action of tensile stress, and type II (slip open type) and type III(tear open type) expand under the act of shear stress. The type I (open type) is most similar to crack propagation in highly brittle materials. The stress intensity $\sigma_{\mathrm{yy}}$ at the crack tip line of type I crack surface displacement is obtained 。

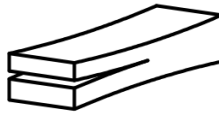

Type I

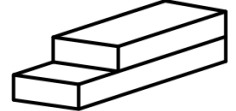

Type II

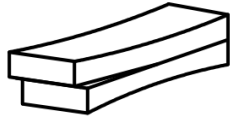

Type III
Fig. 2 Three basic fracture modes

According to Irwin theory, the stress of type I crack in the yy direction is:

$$
\sigma_{y y}=\frac{K}{\left(2 \pi c_{1}\right)^{\frac{1}{2}}} \cos \left(\frac{\theta}{2}\right)\left[1+\sin \left(\frac{\theta}{2}\right) \sin \left(\frac{3 \theta}{2}\right)\right]
$$

When the stress intensity of the crack tip is inert, the crack expands to the ultimate depth inside the workpiece. Under the action of the main cutting force and shear resistance, the crack propagation direction deflects and expands to the workpiece's surface. Combining Eqs. (6) and (7), the crack deflection angle is calculated as:

$$
\cos \left(\frac{\theta}{2}\right)\left[1+\sin \left(\frac{\theta}{2}\right) \sin \left(\frac{3 \theta}{2}\right)\right]=\frac{(2 \pi)^{\frac{1}{2}}}{\psi}
$$

Fig.3 shows a large-scale crack fracture's path. According to the geometric relationship, the crack length $L$ is calculated as:

$$
L=c_{1}+\frac{c_{1} \times \sin \alpha_{1}+h_{\mathrm{D}}}{\sin \alpha_{2}}
$$

Where $h_{D}$ is the actual removal thickness of the material, $\alpha_{1}$ is the first-stage propagation angle, and $\alpha_{2}$ is the second-stage propagation angle of the crack.

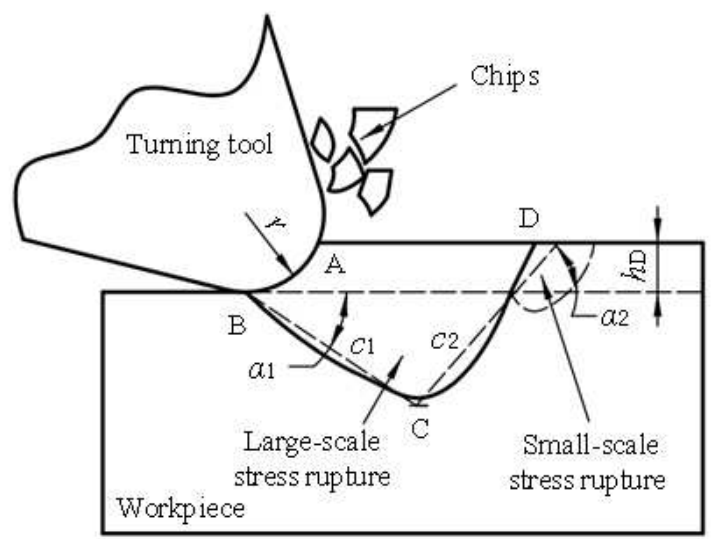


Fig.3. Model of crack system generated by large-scale

$$
\text { stress fracture }
$$

\subsection{Arc radius of tool tip}

In the past, the research on turning mechanisms mainly focused on metal materials. The influence of tool-tip geometry was usually ignored by researchers when selecting the cutting depth in the process of turning. But hard and brittle materials have physical properties such as high hardness, high strength, and high wear resistance. As shown in Fig. 4, in turning to machine, the cutting depth selected is usually far less than the radius of the tool-tip, so the radius of the tool-tip can not be ignored in modeling.

The radius of the arc generated the actual main deviation angle of the tool; it has nothing to do with the calibration of the main deviation angle of the tool and is only related to the

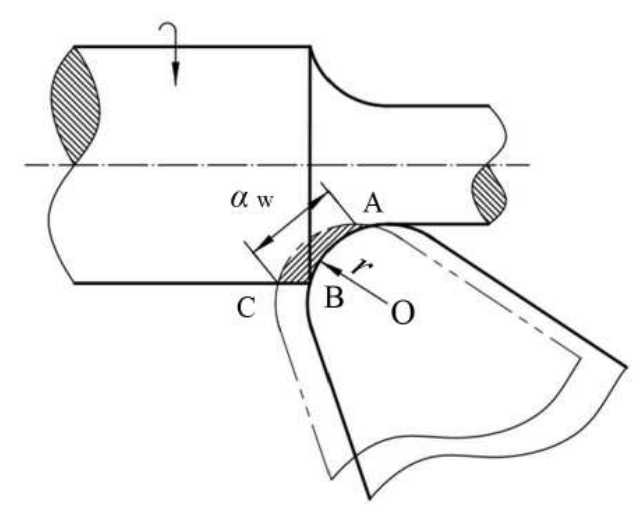

a . Enlarged view of the contact area

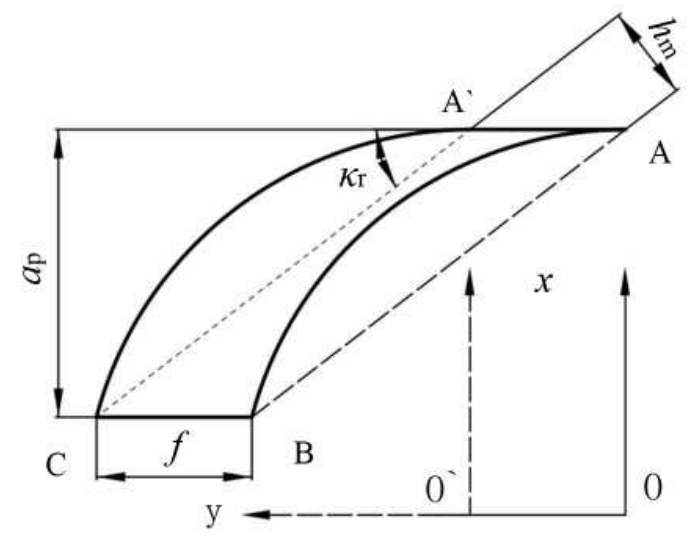

b. Cutting tool - workpiece size area

Fig. 4 Tool - workpiece contact area with tip arc radius radius of the arc of the tool-tip and the depth of cutting. Based on the geometric relation, the main deviation angle $\kappa_{\mathrm{r}}$ is:

$$
\kappa_{\mathrm{r}}=\arcsin \frac{a_{\mathrm{p}}}{\sqrt{2 a_{\mathrm{p}} r}}
$$

The actual cutter-workpiece contact area is a closed area composed of arc $\mathrm{AB}$ and arc $\mathrm{AC}$, and its place is related to the depth of cutting, feed, and the radius of the tool-tip circular arc. The cutting depth and cutting width in the process parameters are not the actual material removal thickness due to the shape change of the tool-workpiece contact zone. In this paper, the depth between the main cutting edge of the turning tool and the free surface of the material is considered the actual material removal thickness. Combining Eq. (10), the actual removal thickness $h_{D}$ of the material is obtained as:

$$
h_{\mathrm{D}}=f \bullet \sin \kappa_{\mathrm{r}}=\frac{f a_{\mathrm{p}}}{\sqrt{2 a_{\mathrm{p}} r}}
$$

In combination with Eq.(10), the geometric relation of the contact zone between the cutter and the workpiece is established, and the actual cutting width $b_{D}$ is obtained:

$$
b_{\mathrm{D}}=\frac{\alpha_{\mathrm{p}}}{\sin \kappa_{\gamma}}=\sqrt{2 a_{\mathrm{p}} r}
$$

\section{The establishment of cutting force theoretical model for turning hard and brittle materials}

Ceramic material is brittle removal, and the formation of fracture chips can be divided into three stages: crack formation, crack propagation, and chip fracture. With the tool feeding, the contact force increases, cracks occur in the elastic-plastic deformation zone, and the strain energy changes. The actual turning process is a constant displacement, and constant force is the loading process, the potential energy is unchanged, the kinetic energy increase. The crack continues to expand beyond 
the unstable state, and the kinetic energy changes. The molecular binding force between the fracture surfaces must overcome when the chips flake off to produce a new surface, and the surface energy changes.

Energy transfer and conversion between tool and workpiece during cutting of hard and brittle materials. According to the law of conservation of energy: tool input is equal to the sum of the energy change between cracks and the work done by the friction force of in turning process. According to Griffith's energy balance theory, the system energy related to crack formation can be divided into mechanical energy and surface energy. Mechanical energy consists of two components: the strain energy stored in the elastic medium and the potential energy provided for external loading.

$$
\begin{aligned}
& U=U_{\mathrm{M}}+U_{\mathrm{S}}+U_{\mathrm{K}} \\
& U_{\mathrm{M}}=U_{\mathrm{E}}+U_{\mathrm{A}}
\end{aligned}
$$

Where, $U$ is Total energy of system, $U_{M}$ is mechanical energy, $U_{S}$ is surface energy, $U_{K}$ is crack kinetic energy, $U_{E}$ is strain energy, $U_{A}$ is potential energy。

\subsection{Energy variation between crack systems}

\subsubsection{Mechanical energy}

The chip element containing the crack can be likened to a cantilever beam as the crack propagates under the push of the cutter, as shown in Fig. 3. The height of the cantilever is approximately equal to the thickness of the cutting layer and the depth of crack propagation. The overhanging length of the cantilever beam is equal to the total length of the crack. In the process of turning, the main driving force of the tool comes from the main cutting force, so the strain energy of the crack system mainly considers the influence of the main cutting force. The strain energy $U_{\mathrm{c}}$ of an elastomer of volume $V$ is as follows:

$$
U_{\mathrm{c}}=\iiint U_{0}(\varepsilon) d V
$$

Where is $U_{0}$ strain energy density and $\xi$ is strain.

The strain energy density depends only on the state of stress:

$$
U_{0}=\frac{\sigma_{\mathrm{F}}^{2}}{E}
$$

According to Griffith equilibrium conditions, the critical conditions for fracture to occur are:

$$
\sigma_{\mathrm{F}}=\frac{1}{Y}\left[2 E \gamma / c_{1}\right]^{\frac{1}{2}}
$$

Where, $Y$ is the geometric factor and $Y=(1 / \pi)^{0.5}$.

According to the geometry of the crack, substituting Eqs.(16) and (17) into Eq.(15), the compressive strain energy is obtained as:

$$
\begin{gathered}
U_{c}=c^{-1} \pi \gamma \sqrt{2 a_{\mathrm{p}} r}\left[\left(\frac{2 a_{\mathrm{p}} f}{\sqrt{2 a_{\mathrm{p}} r}}+c_{1} \sin \alpha_{1}\right) c_{1} \cos \alpha_{1}+\right. \\
\left.\frac{1}{\tan \alpha_{2}}\left(\frac{2 a_{\mathrm{p}} f}{\sqrt{2 a_{\mathrm{p}} r}}+c_{1} \sin \alpha_{1}\right)^{2}\right]
\end{gathered}
$$

The actual turning process is a constant displacement loading process, and the potential energy is zero. Combining Eq. (14), the mechanical energy is obtained as:

$$
U_{\mathrm{c}}=U_{\mathrm{M}}
$$

\subsubsection{The kinetic energy}

As shown in Fig.5, the failure propagation of the crack reaches the failure length, and the material surrounding the rapidly separating crack wall generates kinetic energy due to inertia. Suppose a volume element of unit thickness exists at the point $(x, y)$ in a planar crack system. $\rho \mathrm{d} x \mathrm{~d} y$ is the mass of the volume unit and $\rho$ is the density. At the point $(x, y)$, the displacement component $u_{\mathrm{x}}=u_{\mathrm{x}}(\mathrm{c}, \mathrm{t})$ and $u_{\mathrm{y}}=u_{\mathrm{y}}(x, t)$ 。 Integrate the area $\mathrm{D}$ to obtain the total kinetic energy $U_{\mathrm{K}}$ :

$$
U_{\mathrm{K}}=\frac{1}{2} \rho v^{2} \iint_{\mathrm{D}}\left[\left(\partial u_{\mathrm{x}} / \partial c\right)^{2}++\left(\partial u_{\mathrm{y}} / \partial c\right)^{2}\right] d x d y
$$

To determine the integral value of the kinetic energy integral formula, based on the geometric similarity principle, the void reduction field of the crack should be proportional to the crack length L. $\mathrm{x}, \mathrm{y}$, $\mathrm{ux}$ and uy in the integral term are all proportional to $\mathrm{L}$. The displacement is proportional to the solid, that is, $\sigma \mathrm{u} / \sigma \mathrm{L}$ is proportional to $\sigma \mathrm{a} / \mathrm{E}$. Eq. (20) is simplified as follows: 


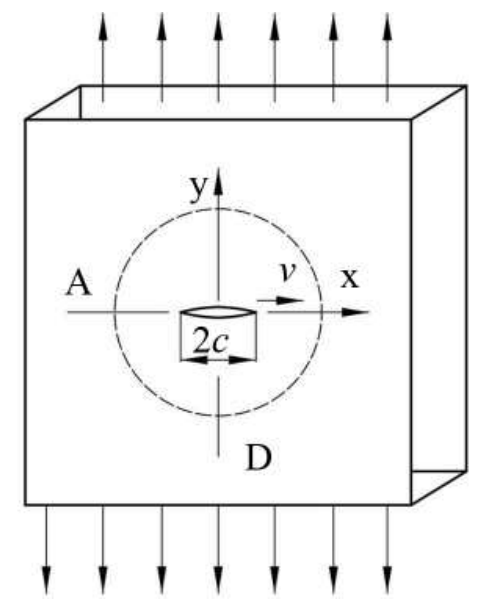

Fig. 5. Dynamic propagation diagram of crack system

$$
U_{\mathrm{K}}=\frac{1}{2} k \rho L^{2} \sigma_{\mathrm{a}}^{2} v_{1}^{2} / E^{2}
$$

Where $v_{\mathrm{t}}=0.38(E / \rho)^{0.5}$ is the limit growth rate of brittle cracks, $L$ refers to the length of cracks produced by the tool-tip pressing the workpiece; $\mathrm{k}$ is a numerical constant.

The external stress acting on the crack surface gradually decreases as the crack grows according to the following flexibility equation:

$$
\sigma_{\mathrm{a}}(c)=\varepsilon_{\mathrm{A}} E /\left[1+\alpha\left(L / L_{0}\right)^{2}\right]
$$

In Eq. (22), L0 refers to the length of the inherent microcrack of the workpiece material; $\xi \mathrm{A}$ is the applied constant strain. The dimensionless parameter $\alpha$ represents the size of the crack relative to the sample size.

As with other solids, the elasticity of ceramics can also be expressed by Hooke's theorem, that is, the relationship between stress and constant strain is:

$$
\varepsilon_{\mathrm{a}}=\sigma_{\mathrm{F}} / E
$$

Incorporate Eqs. (18), (22) and (23) into Eq. (21) to obtain the kinetic energy of the crack thickness B:

$$
U_{\mathrm{K}}=\frac{k \gamma \rho L^{2} v_{1}^{2} B}{c_{1}\left[1+\alpha\left(L / L_{0}\right)^{2}\right]}
$$

Where $\alpha=2 \pi \mathrm{L}_{0}^{2} / \mathrm{A}, \mathrm{A}$ is the area of the pattern on the plane where the crack is located, $\mathrm{A}>>\mathrm{L}_{2}$.

In the turning model, the size of the workpiece is much larger than the crack size, $\alpha \rightarrow 0$. Combining Eqs. (23) and (24), Eq. (24) can be simplified as:

$$
U_{K}=c_{1}{ }^{-1} 0.38^{2} \pi k \gamma L^{2} B
$$

\subsubsection{Surface energy}

When cracks occur in turning materials, the surface area of the system changes from 0 to $2 L B$, and the surface free energy of the cracked system is calculated as:

$$
U_{S}=\int_{0}^{2 L B} \gamma d A=2 L B \gamma
$$

\subsection{The energy input of the tool}

If the cutter has no displacement perpendicular to the direction of the workpiece in one cutting, it considers that the cutting depth resistance does not work. Combine the motion path of the tool and the workpiece, and the motion path of the tool relative to the workpiece is a spiral line. Since the cutting speed is much higher than the feed speed and the work is done by the main cutting force is much greater than the work done by the feed force at the same time. Only the main cutting force is considered in the modeling in this paper, and the work done by the main cutting force is considered as the energy input by the tool. Within the time of largescale crack propagation, the work of the main cutting force is:

$$
W=F_{\mathrm{Z}} v_{\mathrm{c}} t
$$

Where, $t=L / v_{l}$ is the time required for the crack to propagate at the ultimate speed until the length $L$ is reached under ideal conditions (no crack bifurcation or crack stop).

\subsection{Work done by friction}

The cutting deformation coefficient is:

$$
A_{h}=\frac{h_{\mathrm{ch}}}{h_{\mathrm{h}}}=\frac{\cos (\phi-\gamma)}{\sin \phi}
$$

The shear fracture direction of ceramic materials is random. According to statistics, the $\varphi$ should be $45^{\circ}$.

$$
v_{f}=v_{c} \times \frac{\frac{x}{\sin \varphi}}{x \tan \gamma+\frac{x}{\tan \varphi}}=v_{c} \times \frac{\sin \varphi}{\cos (\varphi-\gamma)}
$$

In the process of turning hard and brittle materials, the depth of cutting is constant, and the elastic-plastic deformation of the workpiece is minimal, so the friction between the tool surface and the workpiece is negligible. The work done by the frictional force 
between the tool face and the workpiece is the work done by the frictional force between the tool and the workpiece system.

$$
W_{\mathrm{f}}=F_{\mathrm{f}} v_{\mathrm{f}} t
$$

Combining the vertical Eqs. (26), (27), (28) and (29), the work done by the friction between the toolworkpiece system is as follows:

$$
W_{\mathrm{f}}=F_{\mathrm{z}} v_{\mathrm{c}} t \times \frac{\sin \beta}{A_{\mathrm{h}} \cos (\beta-\gamma)}
$$

\subsection{The establishment of turning force model}

According to the law of conservation of energy, the energy input by the tool is equal to the sum of the energy change between the crack systems and the frictional work. Substituting Eqs.(11), (12), (19), (25), (26), (27) and (31) into Eq. (13), after finishing and simplifying, the main cutting force is:

$$
F_{\mathrm{Z}}=\frac{0.38 v_{\mathrm{c}} \sqrt{E / \rho}}{\left(c_{1}+\frac{c_{1}+h_{\mathrm{D}} \sin \alpha_{1}}{\sin \alpha_{2}}\right)\left(1-\frac{\sin \beta}{A_{\mathrm{h}} \cos (\beta-\gamma)}\right)}\left\{\frac{\gamma \pi \sqrt{2 a_{\mathrm{p}} r}}{c_{1}} \times\right.
$$$$
\left[\left(\frac{f a_{\mathrm{p}}}{\sqrt{2 a_{\mathrm{p}} r}}+\frac{c_{1} \sin \alpha_{1}}{2}\right) c_{1} \cos \alpha_{1}+\frac{1}{\tan \alpha_{2}}\left(\frac{f a_{\mathrm{p}}}{\sqrt{2 a_{\mathrm{p}} r}}+\frac{c_{1} \sin \alpha_{1}}{2}\right)^{2}\right]
$$$$
+0.38^{2} c_{1}^{-1} \pi \gamma k B\left(c_{1}+\frac{h_{\mathrm{D}}+c_{1} \sin \alpha_{1}}{\sin \alpha_{2}}\right)^{2}+2 \gamma \sqrt{2 a_{\mathrm{P}} r}
$$$$
\left.\times\left(c_{1}+\frac{h_{\mathrm{D}}+c_{1} \sin \alpha_{1}}{\sin \alpha_{2}}\right)\right\}
$$

\section{The experiment}

To verify the accuracy of the model, the turning experiment was carried out on the CAK5085 CNC lathe. The material's mechanical properties were as follows: elastic modulus $E=1871 \mathrm{GPa}$, free surface energy per unit area $\gamma=0.30 \mathrm{~J} / \mathrm{m}^{2}$, fracture toughness $K_{\mathrm{IC}}=3.8 \mathrm{MPa} \cdot \mathrm{m}^{1 / 2}$. Density $\rho=2.56 \mathrm{~g} / \mathrm{cm}^{3}$, compressive strength $\sigma_{0}=108 \mathrm{MPa}$. Diameter is $30 \mathrm{~mm}$, length is $100 \mathrm{~mm}$. Machining parameters: cutting speed $v_{\mathrm{c}}=0.32 \mathrm{~m} / \mathrm{min}$, cutting depth $a_{\mathrm{p}}=0.05 \mathrm{~mm}$, feed $f=0.05 \mathrm{~mm}$ 。 Turning experiments were carried out with the tool-tip radius and tool rake Angle as single-factor variables. Table 1 shows the experimental conditions. The experiment of each type of tool parameters is carried out in 6 groups. The cutting force of the turned workpiece is measured by the Kistler 9119AA2 dynamometer, and the average value of the data in the effective phase of the measurement is taken as the result. Fig. 6 shows the cutting force data intercept.

\section{Results and discussion}

\subsection{Machining surface}

The turning tool will not contact the machined surface in a single cutting process. So the surface will retain the crack path of the first stage of large-scale fracture, and the researcher can obtain the initial Angle of crack propagation. As shown in Fig. 7, the OLS4100X 3D laser confocal microscope made several measurements on the machined surface. The average initial crack growth Angle $\alpha_{1}$ in the first stage of large-scale fracture is $23^{\circ}$. After substituting the material properties and the

Table 1 Experimental tool parameters

\begin{tabular}{ccccc}
\hline Experiment number & $\begin{array}{c}\text { Arc radius of tool tip } \\
\mathrm{r}(\mathrm{mm})\end{array}$ & $\begin{array}{c}\text { Anterior horn } \\
\left(^{\circ}\right)\end{array}$ & $\begin{array}{c}\text { Experimental value of cutting } \\
\text { force }\end{array}$ & $\begin{array}{c}\text { Theoretical value of cutting force } \\
\mathrm{F}(\mathrm{N})\end{array}$ \\
\hline 1 & 0.8 & -5 & 26.101 & 24.807 \\
2 & 0.8 & -3 & 24.142 & 23.978 \\
3 & 0.8 & -1 & 24.231 & 23.298 \\
4 & 0.8 & 1 & 23.892 & 22.729 \\
5 & 0.8 & 3 & 22.983 & 22.252 \\
6 & 0.8 & 5 & 22.684 & 21.846
\end{tabular}




\begin{tabular}{ccccc}
7 & 0.4 & -3 & 21.109 & 19.988 \\
8 & 0.6 & -3 & 24.342 & 22.122 \\
9 & 0.8 & -3 & 24.231 & 23.978 \\
10 & 1 & -3 & 27.655 & 25.582 \\
11 & 1.2 & -3 & 28.623 & 27.518 \\
12 & 1.4 & -3 & 29.342 & 28.385 \\
13 & 0.8 & -2 & 23.732 & 23.621 \\
14 & 0.7 & -3 & 23.158 & 23.074 \\
\hline
\end{tabular}

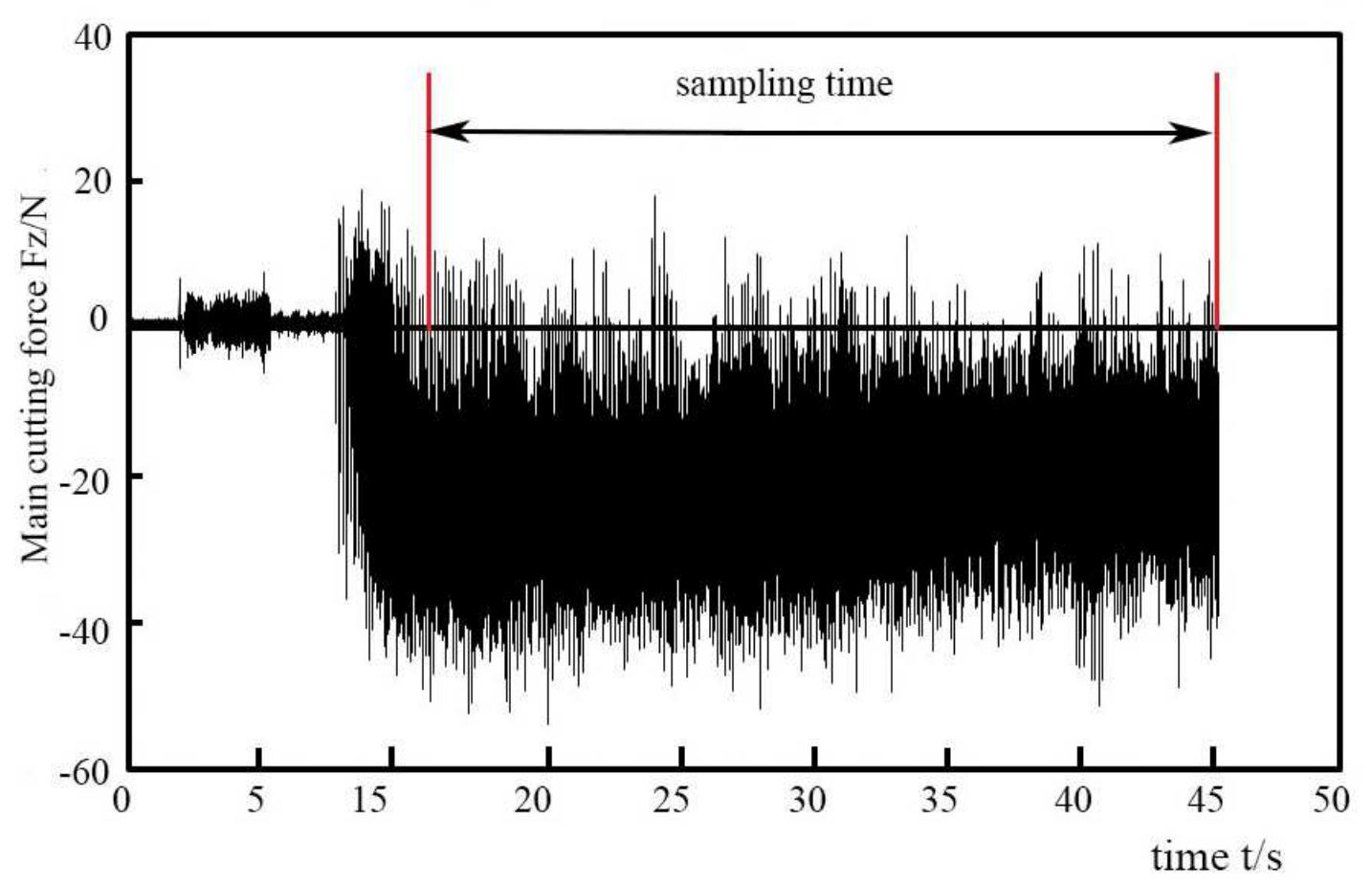

Fig. 6. Dynamic changing process of main cutting force in turning the lithium disilicate glass

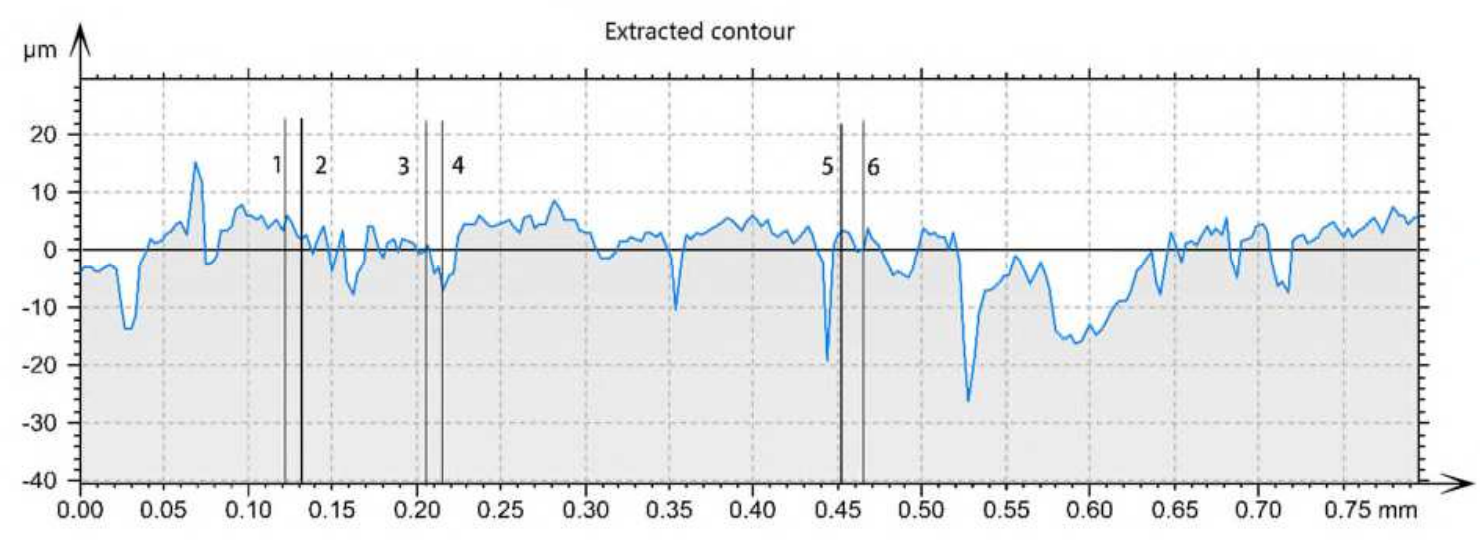

\begin{tabular}{ccccc}
\hline Parameter & Unit & $0-1$ & $2-3$ & $3-4$ \\
\hline Angle & $\circ$ & 21.095 & 27.829 & 20.925 \\
\hline
\end{tabular}

Fig. 7. Cross section profile of machined surface pits 
obtained surface parameters of the pit into Eq. (8), the crack deflection angle $\theta$ is got to be $73^{\circ}$, and the crack growth Angle $\alpha_{2}$ in the second stage is $50^{\circ}$.

\subsection{Influence of tool-tip radius on cutting force}

The influence of tool-tip arc radius on cutting force is shown in Fig. 8. The cutting force increases with the increase of tool tip radius. Ceramic materials have high hardness, high strength, and other physical properties. In the turning process, the cutting depth is much less than the tip radius. When the radius of the tool arc increases, the cutting thickness decreases, and the cutting width increases. As the surface area of the crack increases, the surface energy increases. The energy variation between the cracks increases. The greater the cutter-workpiece contact area, the greater the work done by the friction force. Based on the law of conservation of energy, the energy input of the tool increases. Therefore, the cutting force increases with the expansion of the radius of the tool-tip. The prediction and trend of the model are consistent with the experimental results.

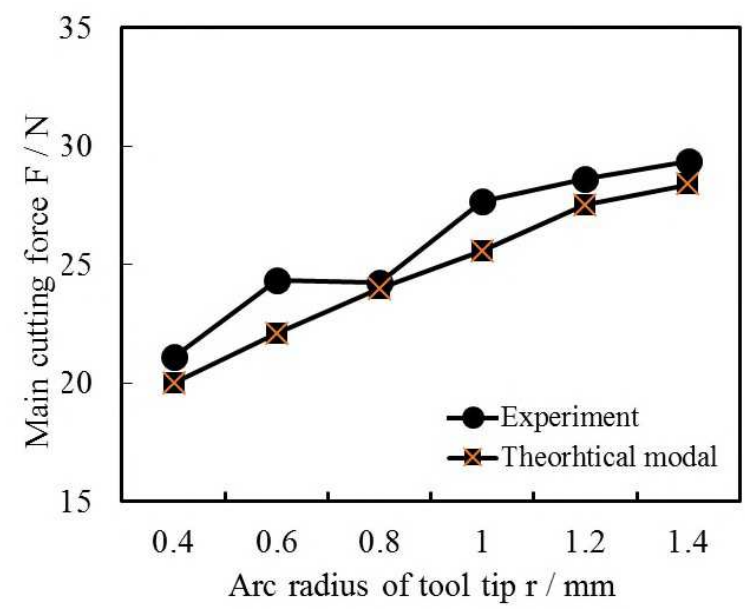

Fig. 8. Variation of main cutting force with tool tip arc

\subsection{Effect of tool rake Angle on cutting force}

In order to study the influence of tool rake Angle on cutting force, the cutting speed $V_{c}=0.5 \mathrm{~mm} / \mathrm{s}$, the feed rate $F=0.05 \mathrm{~mm} / \mathrm{r}$, and the cutting depth $a_{p}=0.05 \mathrm{~mm}$. The influence of rake Angle on cutting force is shown in Fig. 9. As the rake angle of the tool increases, the extrusion of the rake on the workpiece decreases, the friction force do the work between tool and

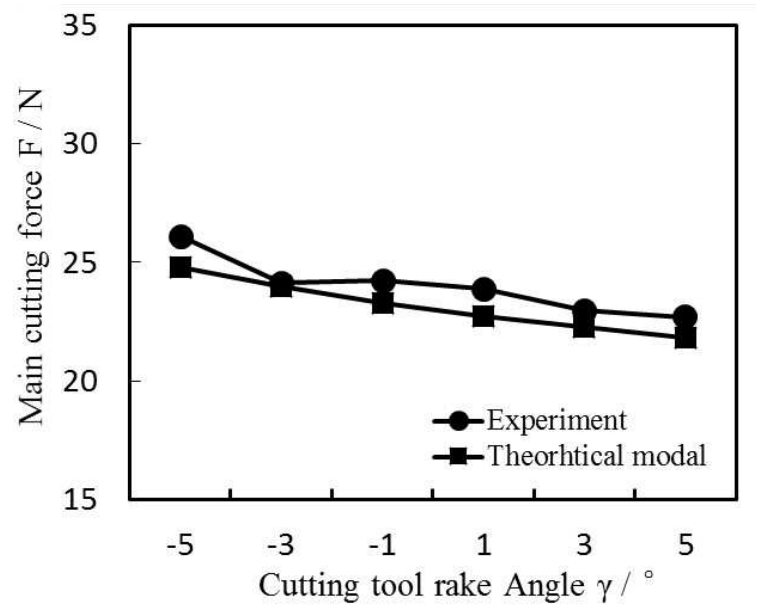

Fig. 9. Variation of main cutting force with tool rake Angle workpiece decreases. According to the principle of conservation of energy, the energy input of the tool is equal to the sum of the work done by friction force and the energy change between the crack system. Under the condition that the three cutting factors remain unchanged, the rake angle of the tool increases, and the cutting force decreases. The prediction and trend of the model are consistent with the experimental results.

\section{Conclusion}

(1) When turning hard and brittle materials, there are two types of fracture removal: large-scale stress fracture and small-scale stress fracture. In the process of turning, the two fracture forms carry out alternately. A large-scale stress fracture is the main cause of force generation and material removal, laying a foundation for the theoretical model of cutting force.

(2) Based on the principle of energy conservation, this paper obtains a theoretical model of cutting force in turning brittle materials by combining the energy change of the crack system during brittle fracture, the work done by the frictional force between the tool and the workpiece, and the total input energy of the tool. The theoretical model shows that the cutting force in turning hard and brittle materials is influenced by the tool's geometrical shape, the material characteristics of the workpiece, and the cutting parameters.

(3) According to the experimental results of turning lithium disilicate ceramics, the main cutting force 
decreases with the increase of the tool raceway. It increases with the rise of tool-tip arc radius.

(4) According to the comparison between the model prediction results and the experimental results, the theoretical values of cutting forces in the theoretical model of turning hard and brittle materials are consistent with the experimental results and agree with the experimental data. Therefore, the theoretical model has high reliability.

\section{Compliance with Ethical}

\section{Standards}

\section{Ethics approval}

This study complies with the ethical standards set out by Springer.

\section{Consent to participate}

All of the authors mentioned in this article participated actively in its development.

\section{Consent for publication}

Yes, consent to publish from all the authors.

\section{Conflict of interest}

The authors declare no competing interests.

\section{Materials availability}

The data and materials set supporting the results are included within the article.

\section{Contributions}

Not applicable.

\section{Funding}

This work was supported by the National Natural Science Foundation of China (grant number 51975113 )

\section{Referernce}

[1] C Li, F Zhang, B Meng, L Liu. Material removal mechanism and grinding force modelling of ultrasonic vibration assisted grinding for $\mathrm{SiC}$ ceramics[J]. Ceramics International,2017,43(3). 2981-2993

[2] Soleimanimehr H, Nategh MJ. An investigation on the influence of cutting-force's components on the work-piece diametrical error in ultrasonic-vibration-assisted turning[C]// INTERNA TIONAL CONFERENCE ON
ADV ANCES IN MATERIALS AND PROCESSING TECHNOLO-GIES (AMPT2010). AIP Publis-hing , (2011),1315(1):1145-1150

[3] LJ Ma, C Li, J Chen, W Li. Prediction model and simulation of cutting force in turning hard-brittle materials[J]. The International Journal of Advanced Manufacturing Technology,2017,91(1-4). 165-174

[4] LJ Ma, A Yu, J Chen. Theoretical model of cutting force in turning the lithium disilicate glass-ceramic[J]. The International Journal of Advanced Manufacturing Technology,2017,92(9-12).

[5] LJ Ma, P He, Hang Deng, T Liu. Theoretical model of characteristic temperature and continuous fracture mechanism of brittle material in the process of turning fluorophlogopite ceramics[J]. Ceramics International,2019,45(9). 429-436

[6] C Zhang, J Zhang, P Feng. Mathematical model for cutting force in rotary ultrasonic face milling of brittle materials[J]. The International Journal of Advanced Manufacturing Technology, 2013,69(1-4). ) 161-170

[7] Z Yang, L Zhu, B Lin. The grinding force modeling and experimental study of $\mathrm{ZrO} 2$ ceramic materials in ultrasonic vibration assisted grin-ding $[\mathrm{J}]$. Ceramics Internationanl, 2019,45(7).8873-8889.

[8] C Zhang, P Feng, Z Wu. Journal of Mechanical Engineering,2011,47(15):149-155. (in Chinese)

[9] Y Cheng, Q Yuan, B Zhang, Z Wang. Study on turning force of $\gamma$-TiAl alloy[J]. The International Journal of Advanced Manufacturing Technology,2019,105(5-6).

[10] Brian Lawn, Rodney Wilshaw. Indentation fracture: principles and applications[J]. Journal of Materials Science,1975,10(6).1049-1081.

[11] Brian R. Lawn. Indentation of Ceramics with Spheres: A Century after Hertz[J]. Journal of the American Ceramic Society,1998,81(8).1977-1994. 
Figures

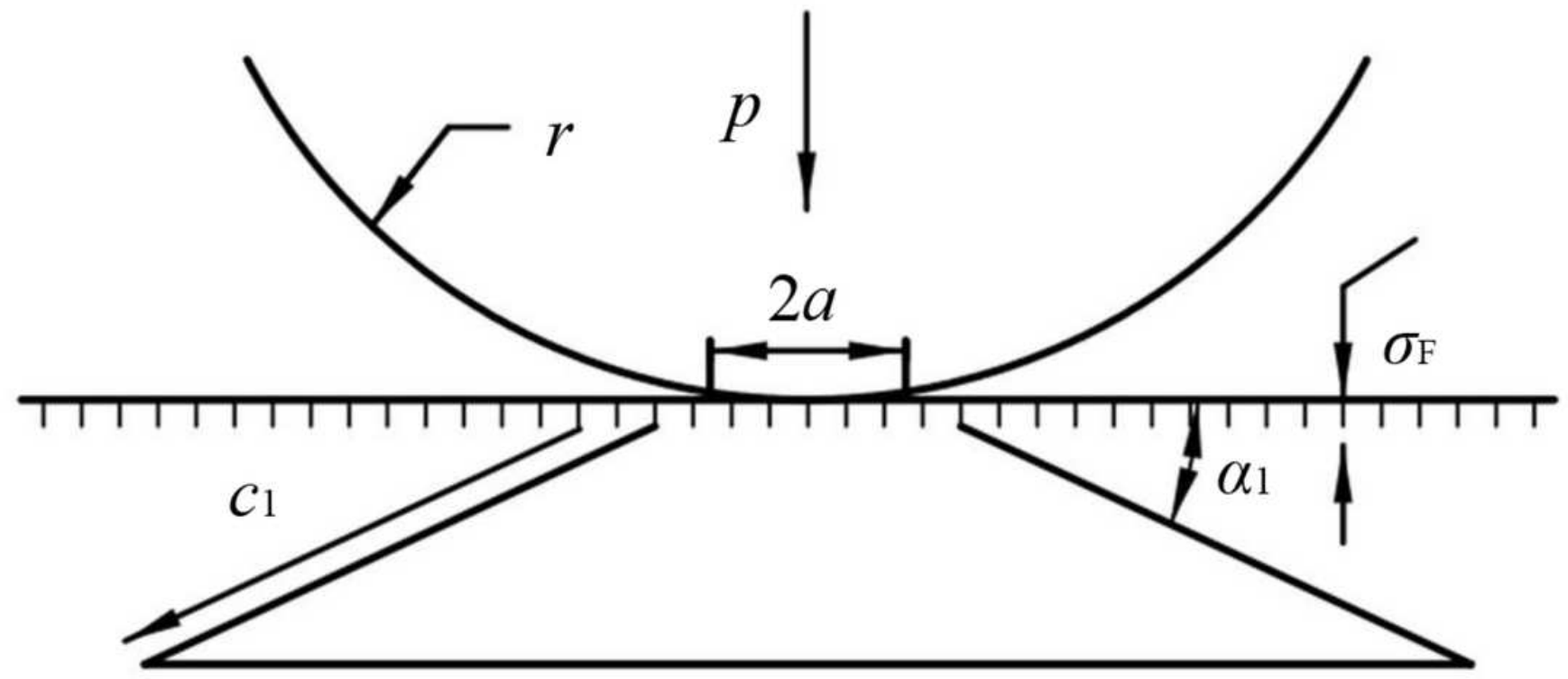

Figure 1

Conical crack system of lithium disilicate ceramics
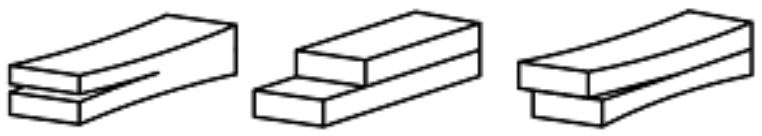

Type I

Type II

Type III

Figure 2

Three basic fracture modes 


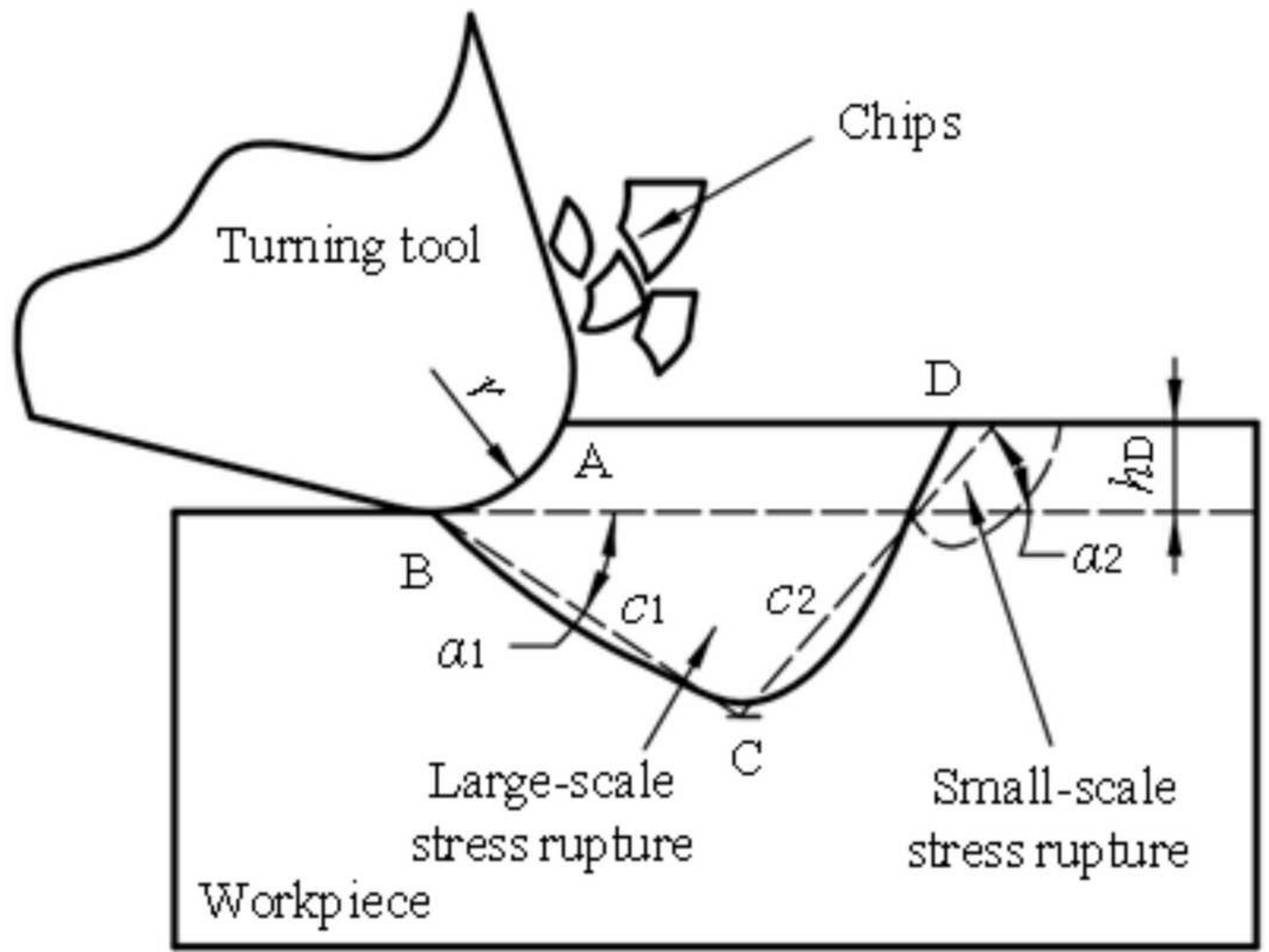

Figure 3

Model of crack system generated by large-scale stress fracture 


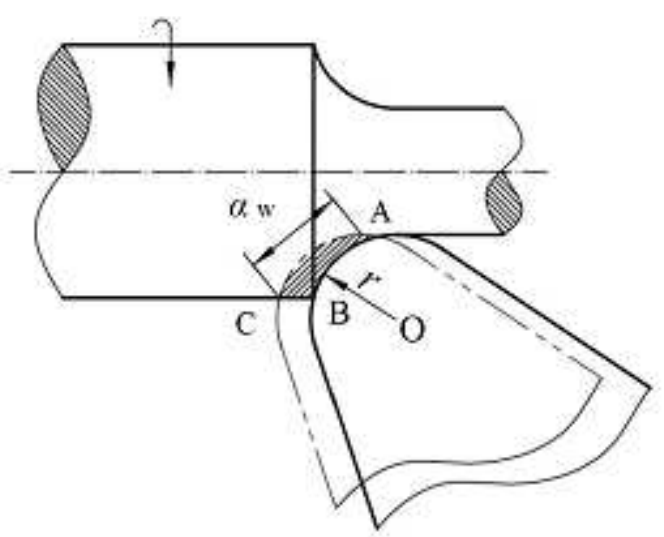

a. Enlarged view of the contact area

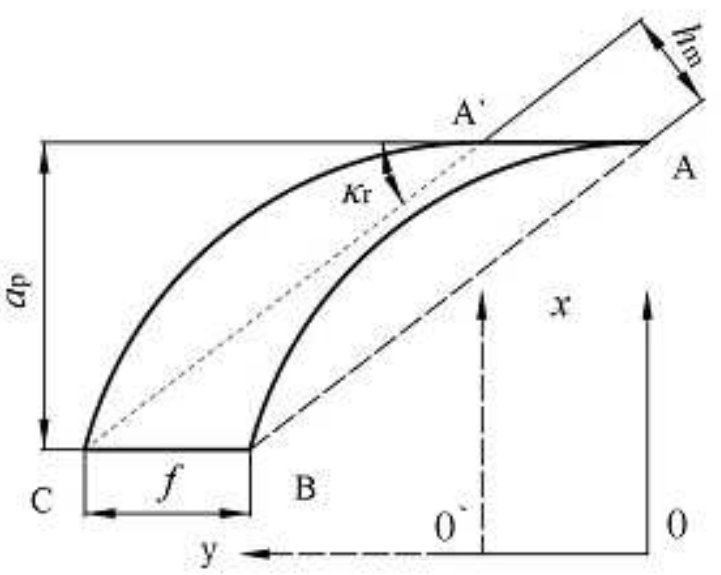

b. Cutting tool - workpiece size area

Figure 4

Tool - workpiece contact area with tip arc radius. 


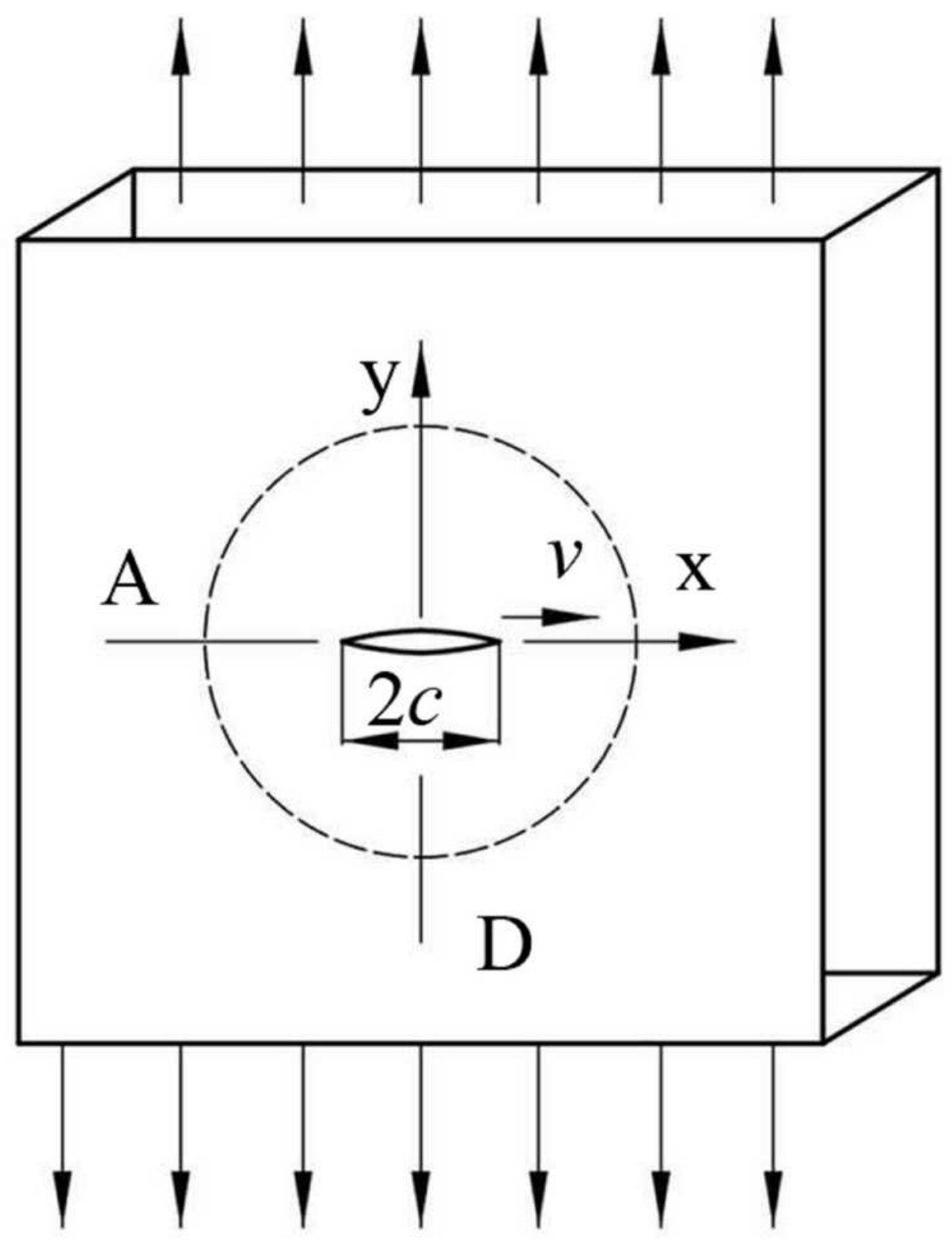

Figure 5

Dynamic propagation diagram of crack system 


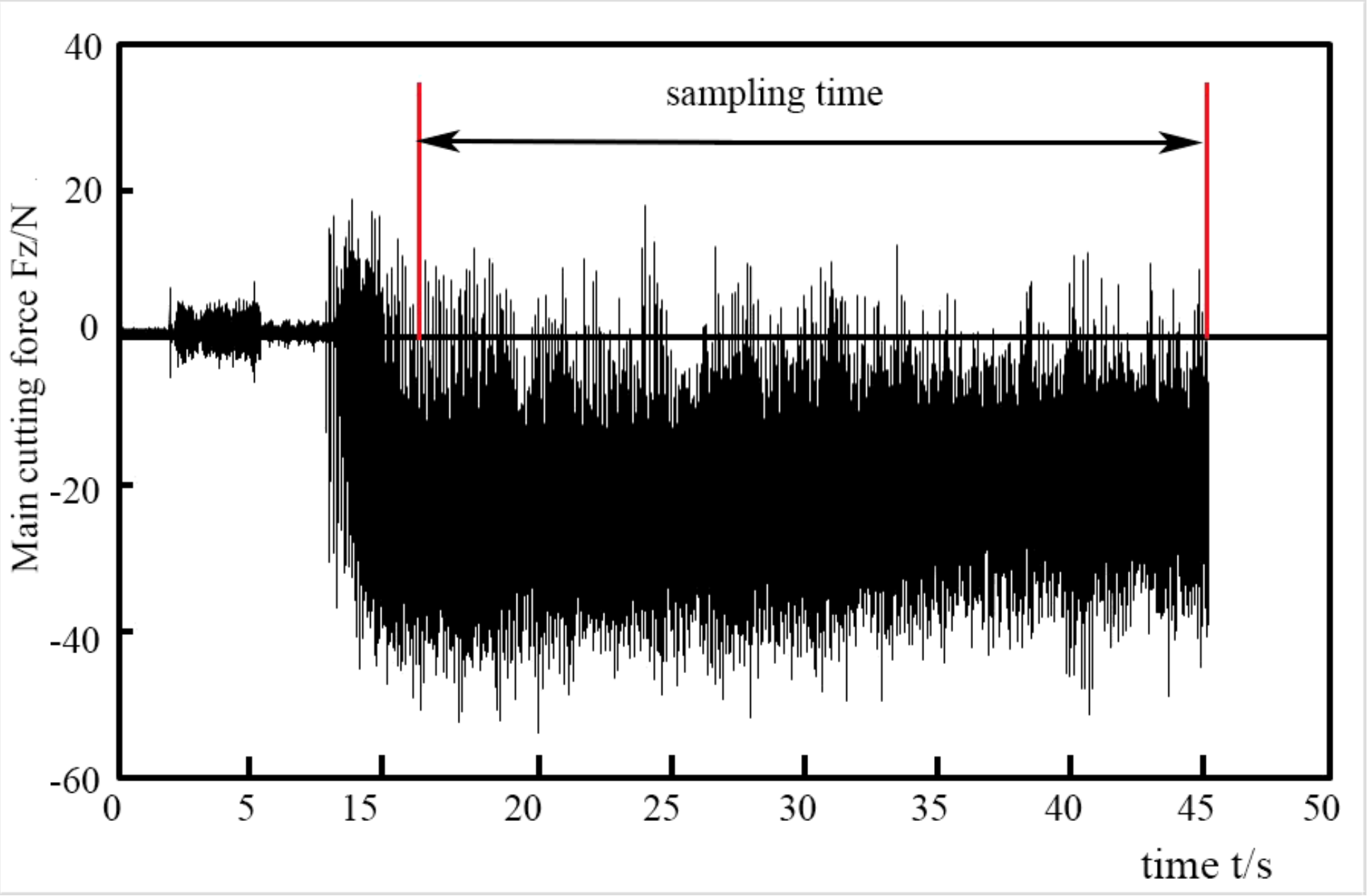

Figure 6

Dynamic changing process of main cutting force in turning the lithium disilicate glass

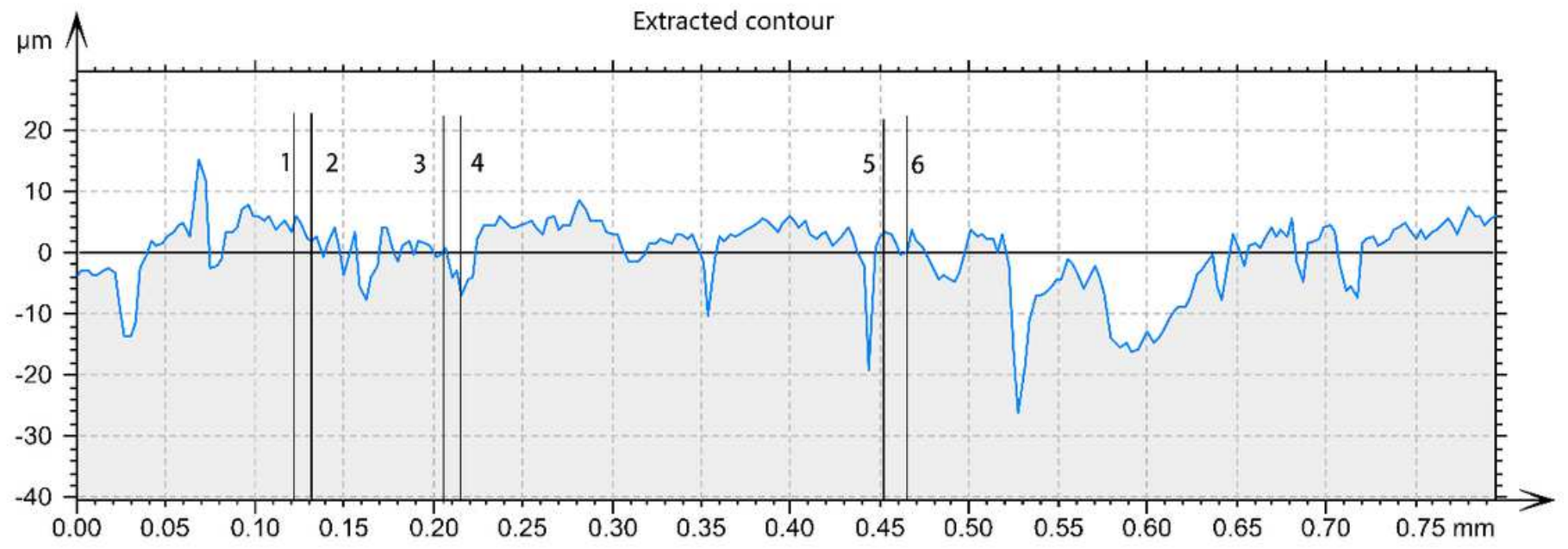

Figure 7

Cross section profile of machined surface pits 


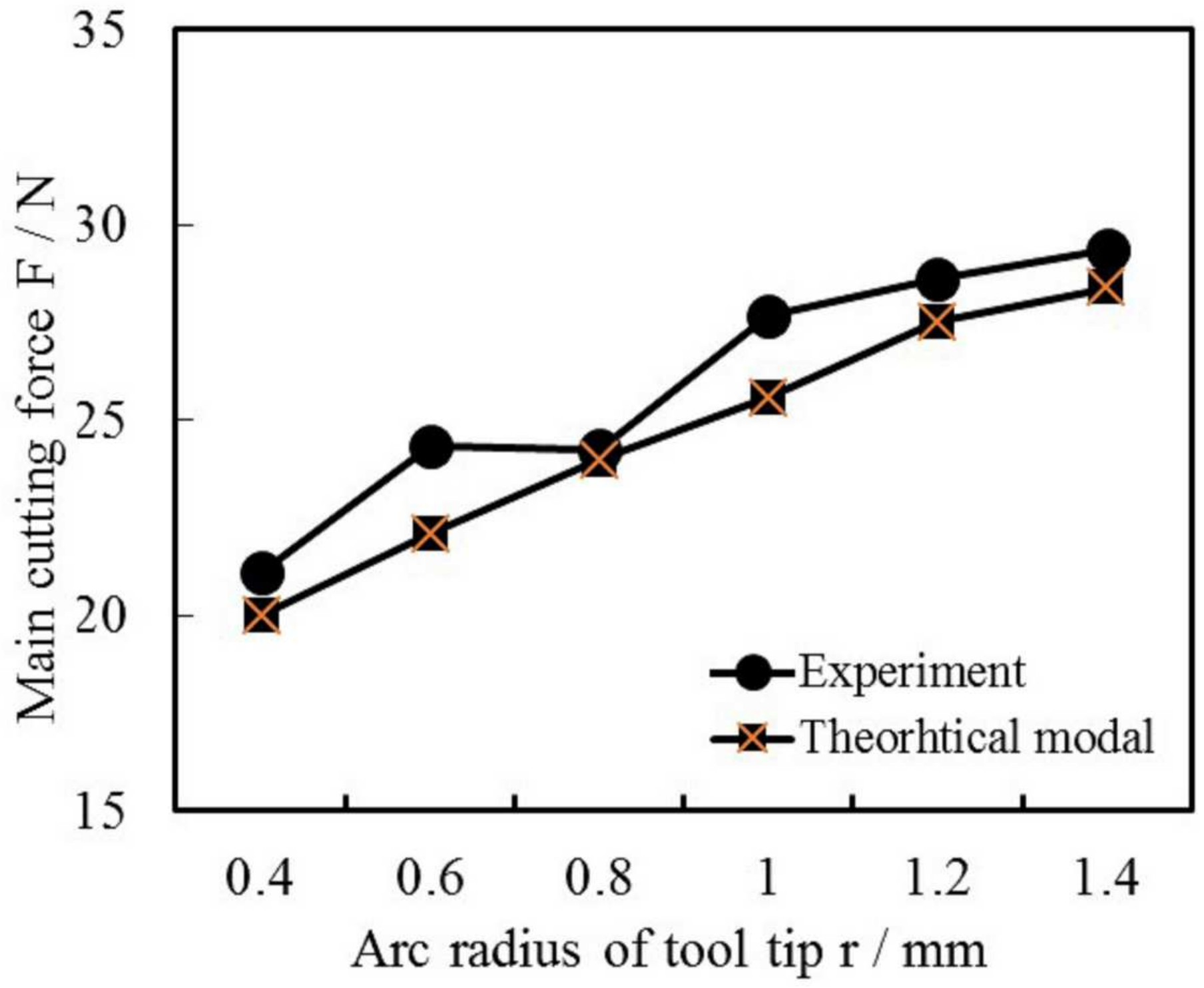

Figure 8

Variation of main cutting force with tool tip arc 


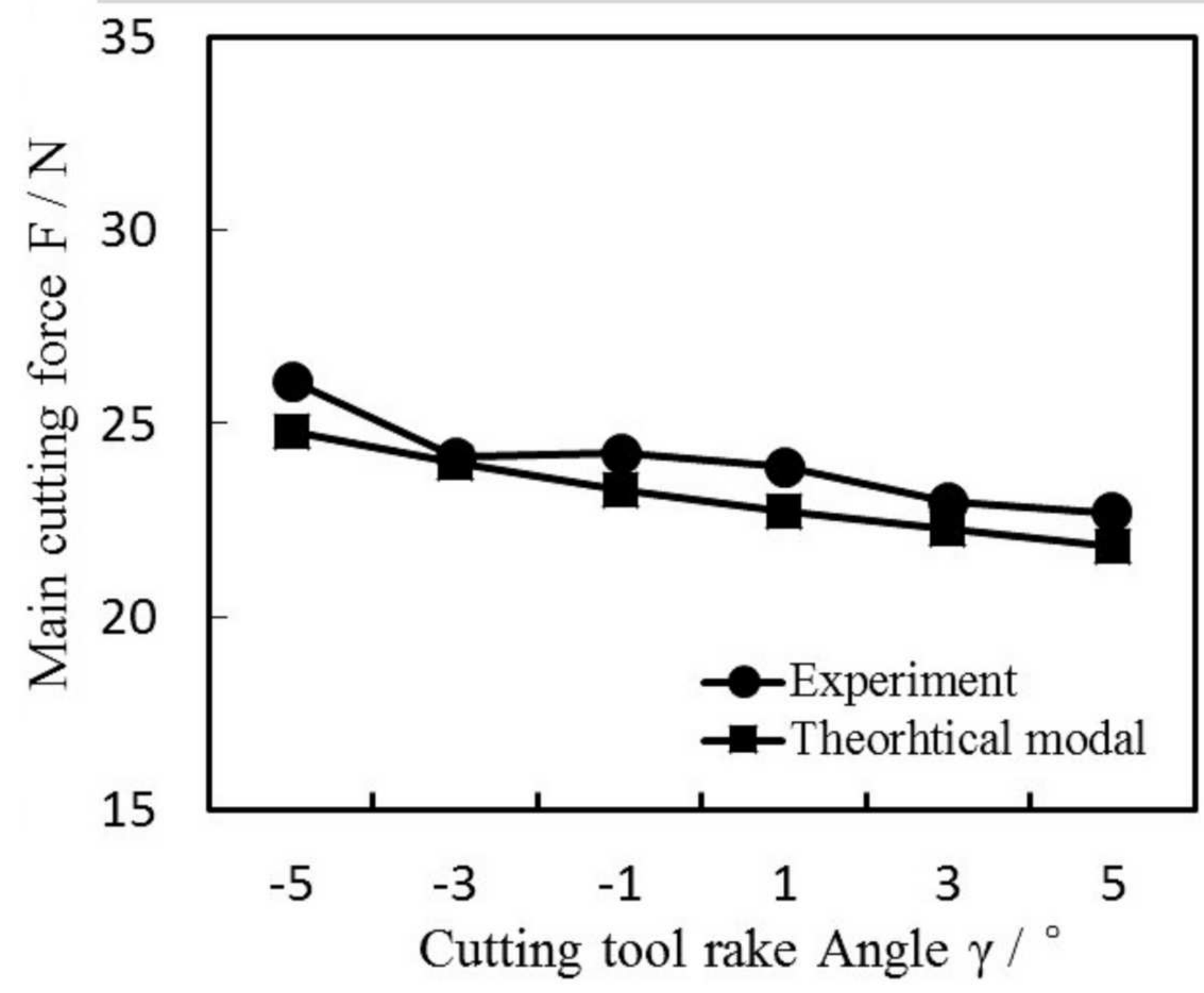

Figure 9

Variation of main cutting force with tool rake Angle 